\title{
Sexual transmission of SARS-CoV-2 virus and its role in the spread of COVID-19: A living systematic review protocol
}

AUTHORS:

Giuliano Duarte

ORCID: 0000-0001-9724-0796

Faculty of Medical Sciences, Midwifery School, Universidad de Santiago de Chile, Santiago, Chile

\section{Luis Ortiz-Muñoz}

ORCID: 0000-0001-6449-2153

UC Evidence Center, Cochrane Chile Associated Center, Pontificia Universidad Católica de Chile, Santiago, Chile

\section{María Belén Morales}

ORCID: 0000-0003-2493-568X

Faculty of Medicine, School of Medicine, Pontificia Universidad Católica de Chile, Santiago, Chile

\section{María Paz Acuña}

ORCID: 0000-0001-7003-495X

Infectious Diseases Unit, Hospital Dr Sótero del Río, Santiago, Chile

Infectious Diseases Unit, Hospital Clinico Dra Eloísa Díaz, La Florida, Santiago, Chile

\section{Gabriel Rada}

ORCID: 0000-0003-2435-0710

Epistemonikos Foundation, Santiago, Chile

UC Evidence Center, Cochrane Chile Associated Center, Pontificia Universidad Católica de Chile, Santiago, Chile

Internal Medicine Department, Faculty of Medicine, Pontificia Universidad Católica de Chile, Santiago, Chile

\section{COVID-19 L·OVE Working Group}

\section{Corresponding author:}

\section{Giuliano Duarte}

Email address: giuliano.duarte@usach.cl

Postal address: El Belloto 3556, Estación Central, Santiago, Chile.

\section{ABSTRACT}




\section{Objective}

To evaluate the presence and duration of SARS-CoV-2 in genital fluids, and determine the risk of sexual transmission of the SARS-CoV-2 virus.

\section{Design}

This is the protocol of a living systematic review.

\section{Data sources}

We will conduct searches in PubMed/Medline, Embase, Cochrane Central Register of Controlled Trials (CENTRAL), grey literature and in a centralised repository in L. OVE (Living OVerview of Evidence). $\mathrm{L} \cdot \mathrm{OVE}$ is a platform that maps PICO questions to evidence from Epistemonikos database. In response to the COVID-19 emergency, L.OVE was adapted to expand the range of evidence it covers and customised to group all COVID-19 evidence in one place. The search will cover the period until the day before submission to a journal.

\section{Eligibility criteria for selecting studies and methods}

We adapted an already published common protocol for multiple parallel systematic reviews to the specificities of this question. We will include randomized trials evaluating the sexual transmission of SARS-CoV-2 virus. Randomised trials evaluating the sexual transmission of other coronaviruses, such as MERS-CoV and SARS-CoV, and non-randomised studies in COVID-19 will be searched in case no direct evidence from randomised trials is found, or if the direct evidence provides low- or very lowcertainty for critical outcomes.

Two reviewers will independently screen each study for eligibility, extract data, and assess the risk of bias. We will perform random-effects meta-analyses and use GRADE to assess the certainty of the evidence for each outcome.

A living, web-based version of this review will be openly available during the COVID-19 pandemic. We will resubmit it if the conclusions change or there are substantial updates.

\section{Ethics and dissemination}

No ethics approval is considered necessary. The results of this review will be widely disseminated via peer-reviewed publications, social networks and traditional media.

\section{PROSPERO Registration}

Submitted to PROSPERO (CRD42020189368).

\section{Keywords}

COVID-19, severe acute respiratory syndrome coronavirus 2, Coronavirus Infections, Systematic review, sexual transmission, STI, sexually transmitted infections.

\section{INTRODUCTION}

COVID-19 is an infection caused by the SARS-CoV-2 coronavirus [1]. It was first identified in Wuhan, China, on December 31, 2019 [2]; three months later, almost half a million cases of contagion had been identified across 197 countries [3]. On March 11, 2020, WHO characterised the COVID-19 outbreak as a pandemic [1]. 
While the majority of cases result in mild symptoms, some might progress to pneumonia, acute respiratory distress syndrome and death [4],[5],[6]. The case fatality rate reported across countries, settings and age groups is highly variable, but it would range from about $0.5 \%$ to $10 \%$ [7]. In hospitalized patients it has been reported to be higher than $10 \%$ in some centres [8].

The SARS-CoV-2 infection, which causes the COVID-19 disease, is mainly transmitted through respiratory droplets and direct contact. The virus has been isolated in different body fluids, such as saliva, faeces, and urine. However, its presence in semen or vaginal fluid, and the role of sexual transmission is unknown.

It's important to know if the SARS-CoV-2 virus is transmitted sexually, since it would allow to implement and reinforce preventive measures of STIs to reduce the spread of the COVID-19 pandemic.

Using innovative and agile processes, taking advantage of technological tools, and resorting to the collective effort of several research groups, this living systematic review aims to provide a timely, rigorous and continuously updated summary of the evidence available on the sexual transmission of the SARS-CoV2 virus.

\section{METHODS}

\section{Protocol and registration}

This manuscript complies with the 'Preferred Reporting Items for Systematic reviews and Meta-Analyses' (PRISMA) guidelines for reporting systematic reviews and meta-analyses [9].

A protocol stating the shared objectives and methodology of multiple evidence syntheses (systematic reviews and overviews of systematic reviews) to be conducted in parallel for different questions relevant to COVID-19 was published elsewhere [10]. This protocol was adapted to the specificities of the question assessed in this review and submitted to PROSPERO (CRD42020189368).

\section{Search strategies}

\section{Electronic searches}

Our literature search was devised by the team maintaining the L.OVE platform (https://app.iloveevidence.com), using the following approach:

1. Identification of terms relevant to the population and exposure components of the search strategy, using Word2vec technology [11] to the corpus of documents available in Epistemonikos Database.

2. Discussion of terms with content and methods experts to identify relevant, irrelevant and missing terms.

3. Creation of a sensitive boolean strategy encompassing all of the relevant terms.

4. Iterative analysis of articles missed by the boolean strategy, and refinement of the strategy accordingly.

Our main search source will be Epistemonikos database (https://www.epistemonikos.org), a comprehensive database of systematic reviews and other types of evidence [12]. We supplemented it with articles from multiple sources relevant to COVID-19 (without any study design, publication status or language restriction) In sum, Epistemonikos Database acts as a central repository. Only articles fulfilling Epistemonikos criteria 
are visible by users. The remaining articles are only accessible

for members of COVID-19 L. OVE Working Group.

Additional searches will be conducted using highly sensitive searches in PubMed/MEDLINE, the Cochrane Central Register of Controlled Trials (CENTRAL), Embase and the WHO International Clinical Trials Registry Platform, without any language or publication status restriction. The searches will cover from the inception date of each database until the day before submission.

The following strategy will be used to search in Epistemonikos Database. We will adapt it to the syntax of other databases.

(coronavir* OR coronovirus* OR "corona virus" OR "virus corona" OR "corono virus" OR "virus corono" OR hcov* OR "covid-19" OR covid19* OR "covid 19" OR "2019-nCoV" OR cv19* OR "cv-19" OR "cv 19" OR "n-cov" OR ncov* OR "sars-cov-2" OR "sars-cov2" OR (wuhan* AND (virus OR viruses OR viral) OR coronav*) OR (covid* AND (virus OR viruses OR viral)) OR "sars-cov" OR "sars cov" OR "sarscoronavirus" OR "severe acute respiratory syndrome" OR "mers-cov" OR "mers cov" OR "middle east respiratory syndrome" OR "middle-east respiratory syndrome" OR "covid-19-related" OR "SARS-CoV-2related" OR "SARS-CoV2-related" OR "2019-nCoV-related" OR "cv-19-related" OR "n-cov-related") AND ((sex* OR intercourse* OR semen* OR vagin*) AND (transmi* OR route* OR source* OR acquisition* OR spread*))

\section{Other sources}

In order to identify articles that might have been missed in the electronic searches, we will do the following:

1. Screen the reference lists of other systematic reviews, and evaluate in full text all the articles they include.

2. Scan the reference lists of selected guidelines, narrative reviews and other documents.

3. Conduct cross-citation search in Google Scholar and Microsoft Academic, using each included study as the index reference.

4. Review websites from pharmaceutical companies producing drugs claimed as effective for COVID-19, websites or databases of major regulatory agencies, and other websites specialised in COVID-19.

5. Email the contact authors of all of the included studies to ask for additional publications or data on their studies, and for other studies in the topic.

6. Review the reference list of each included study.

\section{Eligibility criteria}

\section{Types of studies}

We will include all kinds of studies that answer the research question. Information from non-randomized studies will be used if there is no direct evidence from randomized trials (if any) or if the certainty of the evidence for critical outcomes resulting from randomized trials is rated as low or very low, and the certainty provided by -randomized tests are higher than those provided by randomized evidence. [14]. 
We will exclude studies evaluating the effects on animal models or in vitro conditions.

\section{Types of participants}

We will include trials assessing participants with COVID-19, as defined by the authors of the trials. We will not restrict our criteria to any phase of the disease, whether they have active disease or are recovered. If substantial clinical heterogeneity on how the condition was defined is found, we will explore it using a sensitivity analysis.

In case no direct evidence from randomised trials is found, or if the evidence from randomised trials provides low- or very low-certainty evidence for critical outcomes, we will include information from randomised trials evaluating sexual transmission in other coronavirus infections, such as MERS-CoV or SARS-CoV infections [14].

\section{Type of exposure}

The exposure of interest will be body fluids potentially associated with sexual transmission of SARS-CoV2 , such as the presence of the virus in semen, vaginal fluid or other fluids studied. Infections acquired from a sexual partner or through any sexual contact will be included.

Based on the review question, we will not consider a comparison group.

Type of

outcomes

We will not use the outcomes as an inclusion criteria during the selection process. Any article meeting all the criteria except for the outcome criterion will be preliminarily included and evaluated in full text. We used the core outcome set COS-COVID [15], the existing guidelines and reviews and the judgement of the authors of this review as an input to select the primary and secondary outcomes, as well as to decide upon inclusion. The review team will revise this list of outcomes, in order to incorporate ongoing efforts to define Core Outcomes Sets (e.g. COVID-19 Core Outcomes [16].

\section{Primary outcome}

- SARS-CoV-2 sexually transmitted infection.

Secondary outcomes

- Detection of the virus in sexual fluids

Primary and secondary outcomes will be presented in the GRADE 'Summary of Findings' tables, and a table with all the outcomes will be presented as an appendix [17].

Selection

of

studies

The results of the literature search in Epistemonikos database will be automatically incorporated into the L. OVE platform (automated retrieval), where they will be de-duplicated by an algorithm comparing unique identifiers (database ID, DOI, trial registry ID), and citation details (i.e. author names, journal, year of publication, volume, number, pages, article title and article abstract). 
In the L.OVE platform, two researchers will independently

screen the titles and abstracts yielded by the search against the inclusion criteria. We will obtain the full reports for all titles that appear to meet the inclusion criteria or require further analysis to decide on their inclusion.

We will record the reasons for excluding trials in any stage of the search and outline the study selection process in a PRISMA flow diagram adapted for the purpose of this project.

\section{Extraction and management of data}

Using standardised forms, two reviewers will extract data independently from each included study. We will collect the following information: study design, setting, participant characteristics (including disease severity and age) and study eligibility criteria; details about the administered intervention and comparison, including type of sexual relationship, type of fluid and phase of disease (active or recovered); the outcomes assessed and the time they were measured; the source of funding of the study and the conflicts of interest disclosed by the investigators; the risk of bias assessment for each individual study.

We will resolve disagreements by discussion, and one arbiter will adjudicate unresolved disagreements.

\section{Risk of bias assessment}

The risk of bias for each randomised trial will be assessed using a 'risk of bias' tool (RoB 2.0: a revised tool to assess risk of bias in randomised trials) [18]. We will consider the effect of assignment to the intervention for this review. Two reviewers will independently assess five domains of bias for each outcome result of all reported outcomes and time points. These five domains are: bias due to (1) the randomisation process, (2) deviations from intended interventions (effects of assignment to interventions at baseline), (3) missing outcome data, (4) measurement of the outcome, and (5) selection of reported results. Answers to signalling questions and supporting information collectively will lead to a domain-level judgement in the form of 'Low risk of bias', 'Some concerns', or 'High risk of bias'. These domain-level judgements will inform an overall 'risk of bias' judgement for each result. Discrepancies between review authors will be resolved by discussion to reach consensus. If necessary, a third review author will be consulted to achieve a decision.

We will assess their risks of bias with the Risk Of Bias In Non-randomised Studies of Interventions (ROBINS-I), a tool for assessing risk of bias in non-randomised studies of interventions [19]. We will assess the following domains: bias due to confounding, bias in selection of participants into the study, bias in classification of interventions, bias due to deviations from intended interventions (effect of assignment to intervention), bias due to missing data, bias in measurement of outcomes and bias in the selection of the reported result. We will judge each domain as low risk, moderate risk, serious risk, critical risk, or no information, and evaluate individual bias items as described in ROBINS-I guidance. We will not consider time-varying confounding, as these confounders are not relevant in this setting [19]. As we are studying the general population, we will not consider baseline potential confounders.

\section{Measures of treatment effect}

For dichotomous outcomes, we will express the estimate of treatment effect of an intervention as risk ratios (RR) or odds ratios (OR) along with $95 \%$ confidence intervals (CI).

For continuous outcomes, we will use mean difference and standard deviation (SD) to summarise the data using a 95\% CI. Whenever continuous outcomes are measured using different scales, the treatment effect will be expressed as a standardised mean difference (SMD) with 95\% CI. When possible, we will multiply 
the SMD by a standard deviation that is representative from the

pooled studies, for example, the SD from a well-known scale used by several of the studies included in the analysis on which the result is based. In cases where the minimally important difference (MID) is known, we will also present continuous outcomes as MID units or inform the results as the difference in the proportion of patients achieving a minimal important effect between intervention and control [20]. Then, these results will be displayed on the 'Summary of Findings Table' as mean difference [20].

Strategy for data synthesis

If we include more than one trial we will conduct meta-analysis for studies clinically homogeneous using RevMan 5 [21], using the inverse variance method with random effects model. For any outcomes where data was insufficient to calculate an effect estimate, a narrative synthesis will be presented.

\section{Subgroup and sensitivity analysis}

If relevant heterogeneity is detected, we will perform subgroup analysis according to sex, type of sexual relationship, type of fluid and outcomes. In case we identify significant differences between subgroups (test for interaction $<0.05$ ) we will report the results of individual subgroups separately.

We will perform sensitivity analysis excluding high risk of bias studies, and if non-randomised studies are used, excluding studies that did not report adjusted estimates. In cases where the primary analysis effect estimates and the sensitivity analysis effect estimates significantly differ we will either present the low risk of bias — adjusted sensitivity analysis estimates — or present the primary analysis estimates but downgrading the certainty of the evidence because of risk of bias.

\section{Assessment of certainty of evidence}

The certainty of the evidence for all outcomes will be judged using the Grading of Recommendations Assessment, Development and Evaluation working group methodology (GRADE Working Group) [22], across the domains of risk of bias, consistency, directness, precision and reporting bias. Certainty will be adjudicated as high, moderate, low or very low. For the main comparisons and outcomes, we will prepare Summary of Findings (SoF) tables [20],[23]and also interactive Summary of Findings (http://isof.epistemonikos.org/) tables. A SoF table with all the comparisons and outcomes will be presented as an appendix.

\section{Living \\ evidence \\ synthesis}

An artificial intelligence algorithm deployed in the Coronavirus/COVID-19 topic of the L. OVE platform (https://app.iloveevidence.com/loves/5e6fdb9669c00e4ac072701d) will provide instant notification of articles with a high likelihood to be eligible. The authors will review these and will decide upon inclusion, and will update the living web version of the review accordingly. We will consider resubmission to a journal if there is a change in the direction of the effect on the critical outcomes or a substantial modification to the certainty of the evidence. This review is part of a larger project set up to produce multiple parallel systematic reviews relevant to COVID-19. 


\section{NOTES}

\section{Acknowledgements}

The members of the COVID-19 L.OVE Working Group and Epistemonikos Foundation have made it possible to build the systems and compile the information needed by this project. Epistemonikos is a collaborative effort, based on the ongoing volunteer work of over a thousand contributors since 2012.

\section{Roles}

and

contributions

GR conceived the common protocol for all the reviews being conducted by the COVID-19 L. OVE Working Group. GD drafted the manuscript, and all other authors contributed to it. The corresponding author is the guarantor and declares that all authors meet authorship criteria and that no other authors meeting the criteria have been omitted.

The COVID-19 L. OVE Working Group was created by Epistemonikos and a number of expert teams in order to provide decision makers with the best evidence related to COVID-19. Up-to-date information about the group and its member organisations is available here: epistemonikos.cl/working-group

\section{Competing}

interests All authors declare no financial relationships with any organisation that might have a real or perceived interest in this work. There are no other relationships or activities that could have influenced the submitted work.

\section{Funding}

This project was not commissioned by any organisation and did not receive external funding. Epistemonikos Foundation is providing training, support and tools at no cost for all the members of the COVID-19 L·OVE Working Group.

\section{PROSPERO}

This protocol has been submitted (CRD42020189368).

\section{registration}

\section{Ethics}

As researchers will not access information that could lead to the identification of an individual participant, obtaining ethical approval was waived.

\section{Data}

All data related to the project will be available. Epistemonikos Foundation will grant access to data. 


\section{REFERENCES}

1. WHO2020 World Health Organization. Director-General's remarks at the media briefing on 2019nCoV on 11 February 2020. [Internet] World Health Organization; 2020 [Accessed 2020 April 3]. Available from: https://www.who.int/dg/speeches/detail/who-director-general-s-remarks-at-themedia-briefing-on-2019-ncov-on-11-february-2020

2. Hui2020 Hui DS, I Azhar E, Madani TA, et al. The continuing 2019-nCoV epidemic threat of novel coronaviruses to global health - The latest 2019 novel coronavirus outbreak in Wuhan, China. Int J Infect Dis. 2020 Feb;91:264-266. Available from: doi:10.1016/j.ijid.2020.01.009

3. Dong2020 Dong E, Du H, Gardner L. An interactive web-based dashboard to track COVID-19 in $\begin{array}{llllll}\text { real time. } & \text { Lancet } & \text { Infect } & \text { Dis. } & 2020 & \text { Feb }\end{array}$ Available from: doi:10.1016/S1473-3099(20)30120-1

4. Guang2020 Guan WJ, Ni ZY, Hu Y, et al. Clinical Characteristics of Coronavirus Disease 2019 in China. N Engl J Med 2020. Available from: doi:10.1056/NEJMoa2002032

5. Tavakoli2020 Tavakoli A, Vahdat K, Keshavarz M. Novel Coronavirus Disease 2019 (COVID19): An Emerging Infectious Disease in the 21st Century. BPUMS. 2020;22(6):432-450. Available from: doi:10.29252/ismj.22.6.432

6. Li2020 Li LQ, Huang T, Wang YQ, Wang ZP, Liang Y, Huang TB, et al. 2019 novel coronavirus patients' clinical characteristics, discharge rate and fatality rate of meta-analysis. Journal of medical virology. 2020. Available from: doi:10.1002/jmv.25757

7. CEBM2020 Global Covid-19 Case Fatality Rates [Internet] UK: Centre for Evidence-Based Medicine [Accessed 2020 April 3]. Available from: https://www.cebm.net/covid-19/global-covid19-case-fatality-rates/

8. Rodriguez-Morales2020 Rodriguez-Morales AJ, Cardona-Ospina JA, Gutiérrez-Ocampo E, et al. Clinical, laboratory and imaging features of COVID-19: A systematic review and meta-analysis. Travel medicine and infectious disease. 2020;101623. Available from: doi:10.1016/j.tmaid.2020.101623

9. PRISMA-P Moher D, Shamseer L, Clarke M, et al. Preferred reporting items for systematic review and meta-analysis protocols (PRISMA-P) 2015 statement. Syst Rev. 2015 Jan 1;4:1. Available from: doi:10.1186/2046-4053-4-1

10. PROTOCOLO COMUN MEDWAVE Rada G, Verdugo-Paiva F, Ávila C, Morel-Marambio M, Bravo-Jeria R, Pesce F, et al; COVID-19 L·OVE Working Group. Evidence synthesis relevant to COVID-19: a protocol for multiple systematic reviews and overviews of systematic reviews. Medwave 2020;20(3):e7867. Available from: doi:10.5867/medwave.2020.03.7867

11. Word2vecGithub repository [Internet] [Accessed 2020 April 3]. Available from: https://github.com/dperezrada/keywords2vec

12. EPISTE METHODS Epistemonikos Database Methods [Internet] Santiago: Epistemonikos Foundation [Accessed 2020 April 3]. Available from: https://www.epistemonikos.org/en/about us/methods

13. COVID LOVE METHODS Methods for the special L.OVE of Coronavirus infection [Internet] Santiago: Epistemonikos Foundation [Accessed April 3, 2020]. Available from: https://app.iloveevidence.com/covid-19

14. Schünemann2019 Schünemann HJ, Cuello C, Akl EA, Mustafa RA, Meerpohl JJ, Thayer K, et al; GRADE Working Group. GRADE guidelines: 18. How ROBINS-I and other tools to assess risk 
of bias in nonrandomized studies should be used to rate

the certainty of a body of evidence. J Clin Epidemiol. 2019 Jul;111:105-114. Available from: doi:10.1016/j.jclinepi.2018.01.012

15. COS-COVID Xinyao Jin, Bo Pang, Junhua Zhang, et al. Core Outcome Set for Clinical Trials on Coronavirus Disease 2019 (COS-COVID), Engineering, 2020. Available from: doi:10.1016/j.eng.2020.03.002

16. COVID-19 COS COVID-19 Core Outcomes [Internet]. [Accessed 2020 April 3]. Available from: https://www.covid-19-cos.org/

17. Guyatt2013 Guyatt GH, Oxman AD, Santesso N, et al. GRADE guidelines: 12. Preparing summary of findings tables-binary outcomes. J Clin Epidemiol 2013 Feb;66(2):158-72. Available from: doi:10.1016/j.jclinepi.2012.01.012

18. ROB 2 Sterne JAC, Savović J, Page MJ, Elbers RG, Blencowe NS, Boutron I, et al. RoB 2: a revised tool for assessing risk of bias in randomised trials. BMJ. 2019 Aug 28;366:14898. Available from: doi: $10.1136 / \mathrm{bmj} .14898$

19. ROBINS-I Sterne JA, Hernán MA, Reeves BC, Savović J, Berkman ND, Viswanathan M, et al. ROBINS-I: a tool for assessing risk of bias in non-randomised studies of interventions. BMJ. 2016 Oct 12;355:i4919. Available from: doi:10.1136/bmj.i4919

20. GRADE SOF-CONTINUOS Guyatt GH, Thorlund K, Oxman AD, et al. GRADE guidelines: 13. Preparing summary of findings tables and evidence profiles-continuous outcomes. J Clin Epidemiol 2013 Feb;66(2):173-83. Available from: doi:10.1016/j.jclinepi.2012.08.001

21. RevMan Review Manager (RevMan) [Software]. Version 5.3.5 Copenhagen: The Nordic Cochrane Centre, The Cochrane Collaboration, 2014.

22. GRADE INTRO Guyatt GH, Oxman AD, Vist GE, Kunz R, Falck-Ytter Y, Alonso-Coello P, et al; GRADE Working Group. GRADE: an emerging consensus on rating quality of evidence and strength of recommendations. BMJ. 2008 Apr 26;336(7650):924-6. Available from: doi:10.1136/bmj.39489.470347.AD

23. GRADE SOF-DICOTOMICOS Guyatt GH, Oxman AD, Santesso N, et al. GRADE guidelines: 12. Preparing summary of findings tables-binary outcomes. J Clin Epidemiol [Internet] $2013 \mathrm{Feb}$ [Accessed March 26] ;66(2):158-72. Available from: doi:10.1016/j.jclinepi.2012.01.012 